ZOOLOGIA 27 (3): 431-439, June, 2010

doi: $10.1590 /$ S1984-46702010000300016

\title{
On the female of Metagonia taruma (Araneae: Pholcidae), ecology of the pholcid spiders in the Urucu River Basin, Amazonas, Brazil and new records from Brazilian Amazonia
}

\author{
Leonardo S. Carvalho", ${ }^{1,}$ Sidclay C. Dias²; David F. Candiani² \& Alexandre B. Bonaldo² \\ 1 Universidade Federal do Piauí, Campus Amílcar Ferreira Sobral. Rodovia BR 343, km 3.5, Meladão, 64800-000 Floriano, \\ Piauí, Brazil. \\ ${ }^{2}$ Laboratório de Aracnologia, Coordenação de Zoologia, Museu Paraense Emílio Goeldi. Campus de Pesquisa, Avenida \\ Perimetral, 66040-170 Belém, Pará, Brazil. \\ ${ }_{3}^{3}$ Corresponding author. E-mail: carvalho@ufpi.edu.br
}

\begin{abstract}
In this study we describe the unknown female of Metagonia taruma Huber, 2000, which was discovered after sampling in two forest gap types at Porto Urucu (Urucu River Basin, Coari, Amazonas, Brazil), and also provide information on the community ecology and natural history of the sampled species. The female of $M$. taruma is similar to that of M. samiria (Huber, 2000) by having an epigynum with a slightly projecting broad scape with a distal pocket; it differs by the larger pore plates. We collected twelve Pholcidae species at Porto Urucu and M. taruma was the most frequent and abundant. The populations of Carapoia ocaina Huber, 2000 and Mesabolivar aurantiacus (Mello-Leitão, 1930) present homogeneous sex ratios, while M. taruma and Mesabolivar sp. were female biased. Only two species ( $M$. taruma and Mesabolivar sp. ) exhibited differences in abundance in each forest gap type, being higher at the poorly regenerated gaps. Thus, the use of Pholcidae species as ecological indicators is promising. We also present new records, throughout the Amazon Basin, for the Pholcidae species collected at Porto Urucu.
\end{abstract}

KEY WORDS. Forest gaps; natural history; neotropical region; species distribution; taxonomy.

Pholcidae comprises over 1000 species of spiders in about 85 genera (PlatnicK 2009) and its species occur in a variety of habitats worldwide, from rain forests to deserts, and from sea level to over $3500 \mathrm{~m}$ (Huber 2000). The taxonomic and phylogenetic knowledge of these animals has increased considerably over the past few years (e.g. Huber 2000, 2001, 2005a, b, BRuvoMadaric et al. 2005, Astrin et al. 2006, 2007). The New World genera were subject to a recent comprehensive revision (Huber 2000) and further taxonomical additions (Huber \& BRescovit 2003, Huber 2005a, c, Huber \& Wunderlich 2006, Huber et al. 2005a, b, c, Huber et al. 2010, Huber \& Astrin 2009, Astrin et al. 2006, MACHADO et al. 2007a, b, c). Nevertheless, a large number of taxa are yet undescribed, many of them available in South American collections (HUBER 2000). This is the case for Metagonia Simon, 1893, which occurs from Mexico to Central Argentina and is one of the most species-rich pholcid genus in the neotropics, with 81 described species (Platnick 2009). The taxonomic knowledge of Pholcidae gathered to date contrasts with the sparse ecological and biological data available for most of the New World species, although advancements have already been made (e.g. Briceño 1985, Eberhard 1992a, b, Eberhard \& Briceño 1983, 1985, Huber 1994, 1997a, c, d, 1998a, b, Huber \& Eberhard 1997, Japyassú \& Macagnan 2004, Machado et al. 2007a, c, Peretti et al. 2006, Sewlal \& Starr 2008).
In the present paper, the unknown female of Metagonia taruma Huber, 2000 is described and new records of four pholcid species in the Brazilian Amazon are reported. Data on the community ecology, the abundance of pholcid spiders in two different forest gap types, sex ratios, and natural history of pholcid species from Porto Urucu (Urucu River Basin, Coari, Amazonas, Brazil) are presented.

\section{MATERIAL AND METHODS}

Porto Urucu is a 54000 ha petroleum and natural gas production facility on the right margin of the Urucu River, Solimões River basin, Coari municipality, state of Amazonas, Brazil $\left(04^{\circ} 53^{\prime} \mathrm{S}, 6^{\circ} 20^{\prime} \mathrm{W}\right.$, Fig. 1$)$, where several structured inventory initiatives have been carried out to evaluate the environmental impact of such production activities on the forest matrix fauna. The regional climate is classified as "Afi" in the Köppen system, with the rainy season between September and April, and the dry season between May and August (RADAMBRASIL 1978). The main forest formation in Porto Urucu is "terra firme" (upland forest) associated with lowlands and plateaus irrigated by small creeks ("igarapés"), tributaries of the Urucu River (for a description of an area with similar topography, see Ribeiro et al. 1999). Twelve well regenerated forest gaps (Class 


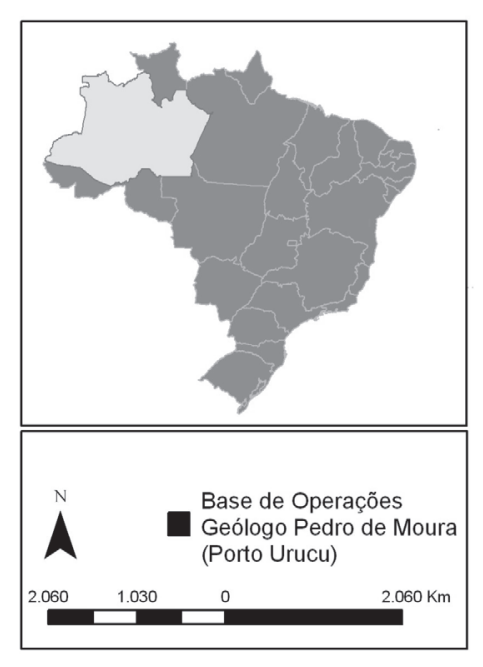

Figure 1. Urucu River Basin, Coari, Amazonas, Brazil.

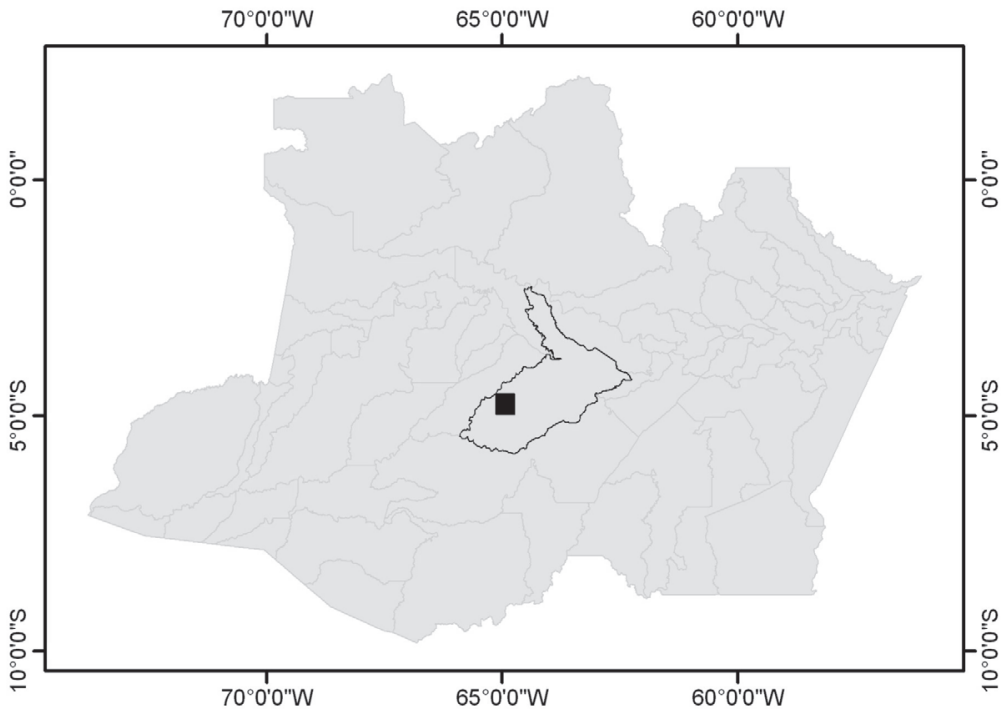

$\stackrel{1}{\circ}$

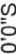

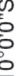

"I-good", age > 5 years) and seventeen poorly regenerated forest gaps (Class "II-poor", age $<5$ years) were sampled during a three month expedition (between June and November, 2006), using Winkler extractors, beating trays, and nocturnal hand collecting. Beating trays were used to access shrub-dwelling spiders during the day. Each tray was composed of a $0.8 \mathrm{~m}^{2}$ white cotton sheet. The nocturnal hand collecting method was modified by combining the "looking up - looking down" methods proposed by CodDington et al. (1991). The samples, combined for each methodology, were the results of one square meter of litter (sifted and placed in Winkler extractors for two days), one hour using beating trays per collector and one hour of nocturnal hand collecting per collector, within an area of $300 \mathrm{~m}^{2}$. To complete the species list, a few individuals gathered on occasional collections were also computed. Kruskal-Wallis tests were used to test if the differences between the abundances by samples for each of the four most abundant species were statistically significant, and a Dunn test was performed to search for the source of statistical differences. Chi-squared tests were used to verify the sex ratio (always expressed as male/female) of each species and its frequency of occurrence (number of samples with a given species divided by the total number of samples with pholcid species). To test statistical differences between the species abundance by the forest gap classes, a MannWhitney test was performed, using all males and females sampled. All analyses were performed using BioEstat 5.0 software (AYres et al. 2007). The statistical analyses were performed at 0.05 levels and only included data for species with abundances higher than ten individuals. The material examined is deposited in the collection of the Museu Paraense Emílio Goeldi, Pará, Brazil (MPEG, curator A.B. Bonaldo). The description style follows Huber (2000). All measurements are in millimeters. The epigynum was dissected and examined in clove oil (Levi 1965), after digestion of soft tissues with pancreatin/borax (ÁlvarezPadilla \& Hormiga 2008). The length/width (L/d) ratio of tibia 1 is a measure of the leg robustness and has a precision of about \pm 2 . The abbreviations used in the text are PME (posterior median eyes) and ALE (anterior median eyes).

\section{RESULTS AND DISCUSSION}

\section{Taxonomy}

\section{Metagonia taruma Huber, 2000}

Figs 2-5

Metagonia taruma Huber, 2000: 61, figs 232-237 (male holotype from “Kuyuwini Landing, Kuyuwini ni river", Upper TakutuUpper Essequibo, Guyana, in American Museum of Natural History, not examined).

Diagnosis. Females of M. taruma differ from the remaining congeneric species, except from Metagonia samiria (Huber, 2000: figs 238-243), by the epigynum with a slightly projecting broad scape with a distal pocket; they differ from $M$. samiria by the larger pore plates (Figs 4-5).

Description. Female (MPEG 13875). Total length 3.1, carapace width 0.9 , length 1.0. Leg I: $23.8(6.2+0.4+5.9+9.5+$ 1.8), tibia II: 3.5 , tibia III: 1.9 , tibia IV: 3.9 , tibia I L/d: 40 . Habitus as in figures 2 and 3. Caparace light ochre, with blackish lines near border and in middle of thoracic region, without thoracic groove, ocular area light ochre, PME-ALE about 20\% of PME diameter. Clypeus and sternum light ochre. Chelicerae ochre. Palps light brown. Legs yellowish, with brown patellae and tibia-metatarsus joints. Tarsus I with more than 20 pseudosegments. Opisthosoma ochre-gray, with some light brown 
spots. Epigynum light brown, with slightly projecting scape and a spoon-like tip; fully symmetrical internally (Figs 4 and $5)$.

Male. Described by Huber (2000: 61). Males collected at Porto Urucu differ slightly with respect to the relative sizes of the distal elements of the procursus, and the clypeus apophysis (the lateral "wings" are more prominent), as described by Huber (2000: 63-64).

Variation. Slight variation in prosoma and opisthosoma coloration pattern with few paler individuals. The carapace of some individuals examined with only scattered blackish lines. Measurements (10 females): total length 3.1-4.0; carapace width 0.8-0.9, length 0.8-1.0; Leg I 3.2-3.7; tibia III 1.8-2.2; tibia IV 3.3-3.8.

Material examined. Brazil, Pará: Juruti (Sítio Barroso, $02^{\circ} 27^{\prime} 41^{\prime \prime S}$, 56 $\left.06^{\circ} 11^{\prime \prime} \mathrm{W}\right), 1$ male, 08.VIII.2004, D.F. Candiani leg. (MPEG 8727); (Vale do Igarapé Mutum, Platô do Rio Juruti, $\left.02^{\circ} 36^{\prime} 11^{\prime \prime S}, 56^{\circ} 12^{\prime} 36^{\prime \prime} \mathrm{W}\right), 1$ female, 04.VIII.2004, D.F. Candiani leg. (MPEG 8790); (Sítio Três Irmãos, 02²7'51.4”S, 5600’08”W), 3 males and 1 female, 08.II.2007, J.A.P. Barreiros leg. (MPEG 13903-13905); 1 female, 16.XI.2007, C. M. Souza leg. (MPEG 13906); Óbidos (Ilha Januaria, Ilha Grande 2, 0206'09"S, 5518’05”W), 4 males, 29.X.2003 (MPEG 1898); Prainha (Margem Norte, Paranaquara, Merendeira, $01^{\circ} 44^{\prime} 38^{\prime \prime}$, $\left.53^{\circ} 10^{\prime} 30^{\prime \prime} \mathrm{W}\right), 3$ males, 21-22.X.2003 (MPEG 1891); (Margem Norte, Fazenda JK, 0151'44"S, 5343'17”W), 1 male, 23.X.2003 (MPEG 1888). Amazonas: Coari (Base de Operações Geólogo Pedro de Moura, Porto Urucu, $\left.04^{\circ} 53^{\prime} \mathrm{S}, 65^{\circ} 20^{\prime} \mathrm{W}\right), 10$ males and 3 females, VIIIX.2006, C.A.C. Santos-Júnior leg (MPEG 13823-13835); 7 males and 14 females, IX-XI.2006, D.F. Candiani leg. (MPEG 1383613852); 3 males, 1 female, VII.2006, L.T. Miglio leg. (MPEG 1385313856); 2 females, X.2006, N.C. Bastos leg. (MPEG 13857, 13858); 10 males, 28 females, IX-X.2006, N.F. Lo-Man-Hung leg. (MPEG 13859-13884); 7 males, 9 females, VII-X.2006, S.C. Dias leg. (MPEG 13885-13899); 1 male, 1 female, 2006, S.C. Dias et al leg. (MPEG 13900); Itacoatiara (Ilha Nossa Senhora do Perpétuo

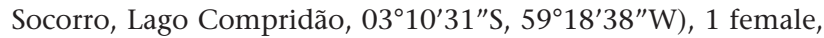
08.XI.2003 (MPEG 1890); (Margem Norte, São José, ilha do Soreano, $03^{\circ} 20^{\prime} 24^{\prime \prime} \mathrm{S}, 58^{\circ} 48^{\prime} 42^{\prime \prime} \mathrm{O}$ ), 3 females, 06.XI.2003 (MPEG 1861); Urucurituba (Ilha Nossa Senhora de Fátima, Ilha Grande, Cucuiari, 02 $\left.46^{\prime} 55^{\prime \prime S}, 57^{\circ} 55^{\prime} 2^{\prime \prime} \mathrm{O}\right), 1$ male, 1 female, 05.XI.2003 (MPEG 1859); 1 male, 05.XI.2003 (MPEG 1893); Urucará (Ilha, Lírio do Vale, Quitéria, $\left.02^{\circ} 23^{\prime} 47^{\prime \prime} \mathrm{S}, 57^{\circ} 30^{\prime} 52^{\prime \prime} \mathrm{O}\right), 3$ females, 03.XI.2003 (MPEG 1860); Parintins (Ilha, Menino de Deus, Catuaba, 02 $2^{\circ} 32^{\prime} 32^{\prime \prime}, 56^{\circ} 32^{\prime} 47^{\prime \prime} \mathrm{W}$ ), 4 females, 01.XI.2003 (MPEG 1858 e 1899).

Distribution. Known from Guyana and Brazil (states of Amazonas and Pará).

\section{New records}

We also collected individuals of four described species, at Porto Urucu River Basin. Below, the records of these species are listed, including our findings and the remaining individuals present in the spider collection of MPEG.

\section{Metagonia beni Huber, 2000}

BRAzIL, Pará: Juruti (Vale do Igarapé Mutum, Platô do Rio Juruti, 02³6'11"S 56²12'36"W), 2 males, 10-13.VIII.2006, D.F. Candiani leg. (MPEG 13901-13902); Óbidos (Ilha, Januaria, Ilha Grande 2, $02^{\circ} 06^{\prime} 09^{\prime \prime} \mathrm{S} 55^{\circ} 18^{\prime} 05^{\prime \prime} \mathrm{W}$ ), 1 female, 29.X.2003 (MPEG 13908); Melgaço (Estação Científica Ferreira Penna, FLONA Caxiuanã, $\left.01^{\circ} 44^{\prime} 18.02^{\prime \prime} \mathrm{S} 51^{\circ} 27^{\prime} 48^{\prime \prime} \mathrm{W}\right), 1$ male, 04.XII.2000, A.B. Bonaldo leg. (MPEG 3517); 1 male, 03.XII.2000, Tommaso leg. (MPEG 3519); Prainha (Margem Sul, Curuarana, Margem do Pixuna, $\left.02^{\circ} 22^{\prime} 58^{\prime \prime S} 54^{\circ} 04^{\prime} 55^{\prime \prime} \mathrm{W}\right), 1$ female, 24.X.2003 (MPEG 1894); Amazonas: Coari (Base de Operações Geólogo Pedro de Moura, Porto Urucu, $04^{\circ} 53^{\prime} \mathrm{S}, 65^{\circ} 20^{\prime} \mathrm{W}$ ), 1 male, 2 females, XI.2006, D.F. Candiani leg. (MPEG 14239, 14241, 14242); 3 males, 3 females, IX-XI.2006, N.F. Lo-Man-Hung leg. (MPEG 14240, 14243-14247).

Distribution. Previously known from Peru (Huánuco, Madre de Dios) and northern Bolivia (Beni). Recorded from Brazil (Amazonas, Pará) for the first time.

\section{Carapoia ocaina Huber, 2000}

Brazil, Pará: Altamira (Novo Progresso, 079953"S, $\left.55^{\circ} 18^{\prime} 53^{\prime \prime} \mathrm{W}\right), 1$ male, 2 females, 19.XI.2005, D.R. Santos-Souza leg. (MPEG 3513); Amazonas: Coari (Base de Operações Geólogo Pedro de Moura, Porto Urucu, $\left.04^{\circ} 53^{\prime} \mathrm{S}, 65^{\circ} 20^{\prime} \mathrm{W}\right), 1$ male, 18.VII.2006 (MPEG 14248); 1 male, IX.2006 (MPEG 14249), C.A.C. Santos-Júnior leg.; 2 males, IX.2006, D.F. Candiani leg. (MPEG 14250-14251); 1 male, 2 females, 2006, S.C. Dias et al leg. (MPEG 14252-14253); 1 male, 2 females, VII.2006, L.T. Miglio leg. (MPEG 14254-14256); 2 females, X.2006, N.C. Bastos leg. (MPEG 14258); 1 female, 19.IX.2006, N.F. Lo-Man-Hung leg. (MPEG 14257); 2 males, 1 female, IX.2006 (MPEG 14259); 2 males, 3 females, X.2006 (MPEG 14260-14263, 14265); 1 male, 1 female, XI.2006 (MPEG 14264); 2 males, IX.2006 (MPEG 14266-14267); 2 males, 1 female, IX.2006, S.C. Dias leg. (MPEG 14269, 14271, 14275); males, 03.IX.2006 (MPEG 14268); 1 male, 11.IX.2006 (MPEG 14274); 1 male, 15.X.2006 (MPEG 14273); 1 male, 1 female, XI.2006 (MPEG 14270, 14272).

Distribution. Previously known from Peru (Amazonas, Huánuco, Loreto) and Brazil (Amazonas: Manaus). Recorded for the first time from the Brazilian municipalities Altamira (State of Pará) and Coari (Amazonas).

\section{Mesabolivar aurantiacus (Mello-Leitão, 1930)}

BraziL, Amapá: Laranjal do Jari (Jari), 2 males, 4 females, (MPEG 3474); 1 female (MPEG 3475), 22-23.II.2003, D.D. Guimarães leg.; 5 males, 3 females, 21-23.II.2003, J.A.P. Barreiros leg. (MPEG 3472, 3476-3480); Pará: Almeirim (Ilha Arumanduba, Genipapo, $\left.01^{\circ} 29^{\prime} 42^{\prime \prime} S, 52^{\circ} 27.23^{\prime} 14^{\prime \prime} \mathrm{W}\right), 1$ male, 2 females, 20.X.2003, T. Gardner \& M.A. Ribeiro-Júnior leg. (MPEG 1887); (Jari, 01 $01^{\circ} 33^{\prime \prime} S, 52^{\circ} 34^{\prime} 02^{\prime \prime} \mathrm{W}$ ), 1 female, 22.II.2003, J.A.P. Barreiros leg. (MPEG 3473); 2 males, 11.II.2005 (MPEG 2657, 2658); 1 male, 18.V.2005 (MPEG 3466); 3 males, 1 female, 22.VI.2005 (MPEG 2654, 2659, 2660); 1 male, 2 females, 25.V.2005 (MPEG 3465, 2656); 1 male, 15.VI.2005 (MPEG 2655); 

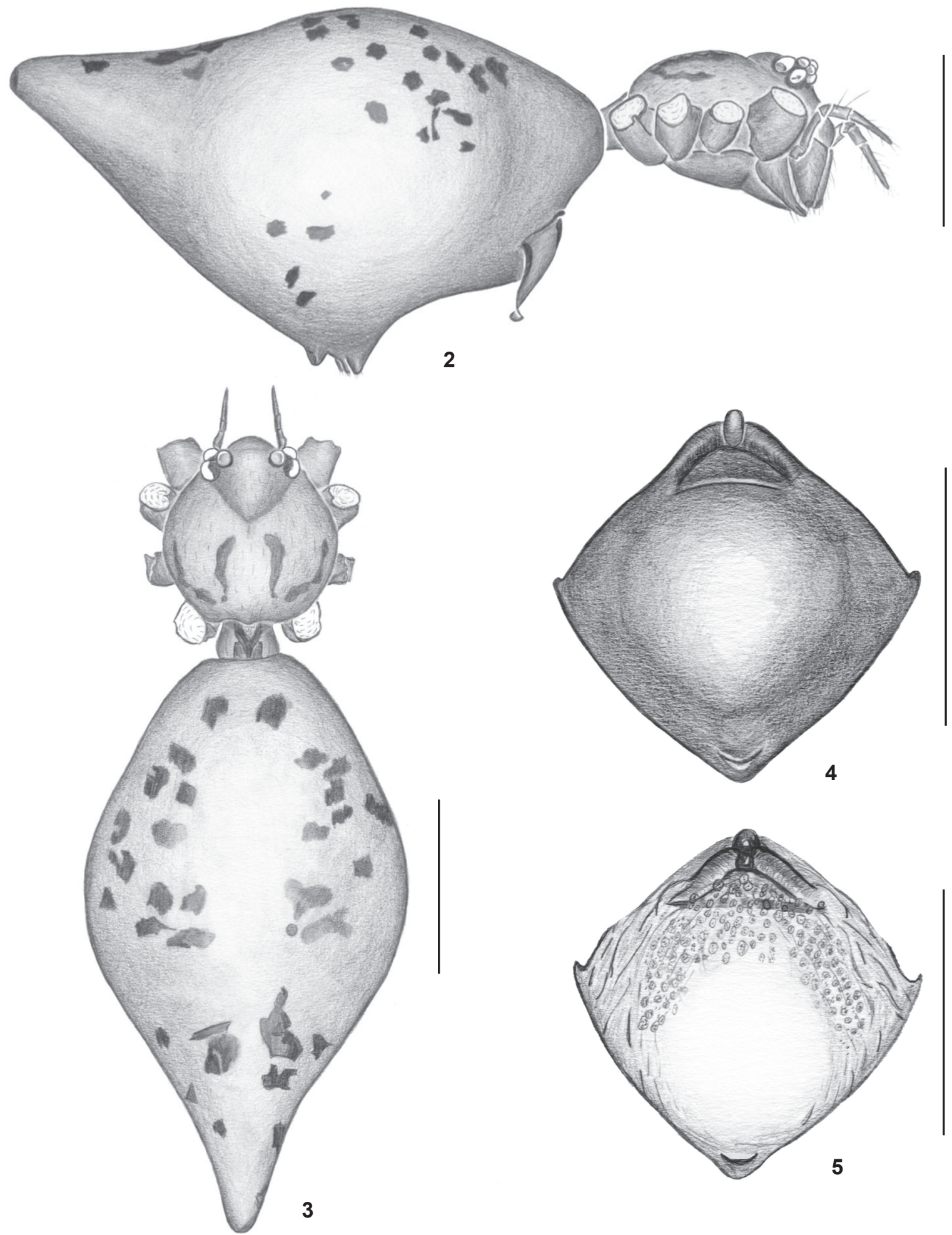

Figures 2-5. Metagonia taruma, female: (2-3) habitus, lateral (2) and dorsal (3) views; (4-5) cleared female genitalia, ventral (4) and dorsal (5) views. Scale bars: (2-3) $1 \mathrm{~mm},(4-5) 0.5 \mathrm{~mm}$. 
5 males, 4 females, VIII-IX.2004, T. Gardner leg. (MPEG 3467 3471); Juruti (Vale do Igarapé Mutum, Platô do Rio Juruti, $\left.02^{\circ} 36^{\prime} 11^{\prime \prime} \mathrm{S}, 56^{\circ} 12^{\prime} 36^{\prime \prime} \mathrm{W}\right), 2$ males, 04.VIII.2004, D.F. Candiani \& D.R. Santos-Souza leg. (MPEG 8737, 8739); 6 males, 1 female, D.R. Santos-Souza leg. (MPEG 8803, 8813); (Sítio Barroso, $\left.02^{\circ} 27^{\prime} 41^{\prime \prime} \mathrm{S}, 56^{\circ} 00^{\prime} 11^{\prime \prime} \mathrm{W}\right), 2$ males, 08.VIII.2004, D.R. SantosSouza leg. (MPEG 8812); (Sítio Três Irmãos, 02²7'51"S, $\left.56^{\circ} 00^{\prime} 08^{\prime \prime} \mathrm{W}\right), 3$ males, 3 females, 04.III.2006, D.R. Santos-Souza leg. (MPEG 9101, 9106); Platô Capiranga, Linha 168E, $\left.02^{\circ} 28^{\prime} 22^{\prime \prime} \mathrm{S}, 56^{\circ} 12^{\prime} 29^{\prime \prime} \mathrm{W}\right), 1$ male, 1 female, 11.III.2006, D.R. Santos-Souza leg. (MPEG 9208); 3 males, 4 females, 08.III.2006, D.R. Santos-Souza leg. (MPEG 9207); Senador José Porfírio (Rio Xingu), 1 male, 03.III.2001 (MPEG 2341); Altamira (Castelo de Sonhos, $08^{\circ} 20^{\prime} 03^{\prime \prime}$ S, 55 $\left.12^{\prime} 52^{\prime \prime} \mathrm{W}\right), 1$ female, 17.XI.2005, D.R. Santos-Souza leg. (MPEG 2652); Prainha (Margem Norte,

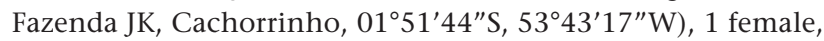
23.X.2003 (MPEG 1883); Santa Isabel (Rio Itá), 1 male, 09.XII.2001, A.B. Bonaldo \& J.A.P. Barreiros leg. (MPEG 2354); Tucuruí (Rio Tocantins), 1 male, 22.VI.1984, W.L. Overal leg. (MPEG 2345); Barcarena (013'소 $\left.48^{\circ} 40^{\prime} \mathrm{W}\right), 1$ male, 20.XI.2001, A.B. Bonaldo leg. (MPEG 2356); 3 males, 3 females, D.D. Guimarães leg. (MPEG 2347, 2357-2358); Santa Bárbara (Vila Denpasa), 1 male, 21.IV.2002, D.R. Santos-Souza leg. (MPEG 2350); Benevides (Fazenda Souza), 2 males, 27.I.2002, D.R. Santos-Souza leg. (MPEG 2351-2352); 1 male, 1 female, 03.IV.2001, D.R. Santos-Souza leg. (MPEG 2353); Santo Antônio do Tauá, 1 male, 02.VIII.2002, L.T. Miglio leg. (MPEG 2344); Amazonas: Tabatinga (Margem Norte, Palmares, Terra Firme Palmares, $\left.04^{\circ} 00^{\prime} 51^{\prime \prime} \mathrm{S}, 69^{\circ} 27^{\prime} 51^{\prime \prime} \mathrm{W}\right), 1$ female, 11.IX.2003 (MPEG 1897); São Paulo de Olivença (Margem Sul, Palmares, Solimões, 03²8'37"S, 69²'59”W), 1 female, 11.IX.2003 (MPEG 1892); Coari (Base de Operações Geólogo Pedro de Moura, Porto Urucu, $\left.04^{\circ} 53^{\prime} \mathrm{S}, 65^{\circ} 20^{\prime} \mathrm{W}\right)$, males, 1 female, 18.VII.2006, C.A.C. Santos-Júnior leg. (MPEG 14277); 2 males, 1 female, IX-X.2006, D.F. Candiani leg. (MPEG 14278-14280); 1 male, VII.2006, L.T. Miglio leg. (MPEG 14281); 6 males, 8 females, IX-X.2006, N.F. Lo-Man-Hung leg. (MPEG 14282-14292); 1 female, 29.VII.2006, S.C. Dias leg. (MPEG 14296); 6 males, 1 female, IX-X.2006, S.C. Dias leg. (MPEG 14293-14295, 14297-14298); Itacoatiara (Ilha Nossa Senhora de Fátima, Ilha do Januário, 03¹0.87'52"S, 59¹8.52' 31”W), 1 male, 08.XI.2003 (MPEG 1884); 1 female, 06.XI.2003 (MPEG 1895); Urucurituba (Ilha Nossa Senhora de Fátima, Ilha Grande Cucuiari, $\left.02^{\circ} 46^{\prime} 18^{\prime \prime} \mathrm{S}, 57^{\circ} 55^{\prime} 42^{\prime \prime} \mathrm{W}\right), 1$ male, 05.XI.2003 (MPEG 1896); Parintins (Ilha Menino de Deus, Ilha do Meio, 02³1'18"S, 56 31'44”W), 1 male, 01.XI.2003 (MPEG 1886), C.A. Rheims leg.; 1 female, 01.XI.2003 (MPEG 1885); (Ilha Menino de Deus, Catuaba, 02 $31^{\prime} 18^{\prime \prime} S, 56^{\circ} 31^{\prime} 44^{\prime \prime} \mathrm{W}$ ), 2 females, 01.XI.2003 (MPEG 1889).

Distribution. Previously known from Surinam, Guyana, Trinidad, Colombia, Ecuador, Peru, Bolivia and Brazil (Amazonas, Pará, Mato Grosso, Acre). Recorded for the first time from the Brazilian State of Amapá and the following municipalities of the State of Pará: Almeirim, Altamira, Barcarena,
Benevides, Juruti, Santa Bárbara, Santa Isabel, Santo Antônio do Tauá, Senador José Porfírio and Tucuruí.

\section{Litoporus dimona Huber, 2000}

BrazIL, Amazonas: Coari (Base de Operações Geólogo Pedro de Moura, Porto Urucu, $\left.04^{\circ} 53^{\prime} \mathrm{S}, 6^{\circ} 20^{\prime} \mathrm{W}\right), 1$ female, IX.2006, C.A.C. Santos-Júnior leg. (MPEG 14234); 1 female, VII.2006, L.T. Miglio leg. (MPEG 14237); 2 males, 1 female, 27.IX.2006, N.F. Lo-Man-Hung leg. (MPEG 14235); 2 males, XI.2006 (MPEG 14236); 2 females, IX.2006, S.C. Dias leg. (MPEG 14238, 14233).

Distribution. Previously known only from the type-locality Dimona Reserve, $80 \mathrm{~km}$ N Manaus, Amazonas, Brazil. Recorded for the first time from Coari, Amazonas, Brazil.

\section{Ecology and natural history}

Combining the material from the two forest gap classes with additional occasional collections, 241 adult pholcid spiders representing eleven species were collected. This represents the highest known pholcid diversity on a single locality in the Brazilian Amazon, surpassing the nine and eight species recorded from Floresta Nacional de Caxiuanã (Bonaldo et al. 2009) and Reserva Florestal Adolpho Ducke (Höfer \& BREsCovit 2001), respectively. On the other hand, for the entire Amazon Basin, the highest Pholcidae species richness is recorded for Pakitza (Madre de Dios, Peru), with twelve species (Silva \& Coddington 1996). Four pholcid species collected at Porto Urucu were singletons belonging to Ibotyporanga Mello-Leitão, 1944 (collected with Winkler extractor), Otavaloa Huber, 2000 species (collected during nocturnal hand searches), and two species of undetermined genera (Pholcidae sp. 1 and sp. 2; both collected with beating trays). Other infrequent species were L. dimona $(\mathrm{n}=9)$, M. beni $(\mathrm{n}=9)$ and an undetermined species of Litoporus Simon, $1893(\mathrm{n}=2)$. The remaining four species, all collected with beating trays and nocturnal hand searches, were abundant at Porto Urucu: M. aurantiacus $(\mathrm{n}=29)$, C. ocaina $(\mathrm{n}=34)$; Metagonia taruma $(\mathrm{n}=98)$ and an undescribed species of Mesabolivar González-Sponga, 1998 ( $\mathrm{n}=56$, B.A. Huber \& E.O. Machado, pers. comm.). Most M. aurantiacus and Mesabolivar sp. specimens were collected in nocturnal hand searches, and for C. ocaina the abundances using both collecting methods were similar.

Species of Metagonia are known to inhabit leaf litter, foliage and caves (Huber 1997a, b, 1998a, Pérez-GonzÁlez \& Huber 1999, Huber et al. 2005c, Huber \& SCHÜtte 2009), and most of the forest species are adapted to a cryptic life on the underside of living leaves, with pale greenish bodies, very thin and long legs, elongated abdomens, and sometimes even elongated eggsacs (Huber et al. 2005c). Metagonia taruma is a foliage-dwelling species with all of these cryptic morphological features, although its egg-sac shape has never been described and neither was it observed in the present study. This species was most efficiently collected with beating trays, a method that easily accesses the herb and shrub layers (Coddington et al. 1991), and can also be found in the forest canopy or sub-canopy, as 
attested by fogging samples at Urucu River Basin, when the shrub layer was removed.

Metagonia taruma was significantly the most abundant (Kruskal-Wallis: $\mathrm{H}=73.4694$; d.f. $=3$; $\mathrm{p}<0.0001$ ) and frequent $\left(\chi^{2}=27.346\right.$, d.f. $\left.=3, p<0.0001\right)$ Pholcidae species from Porto Urucu. The abundance pattern of Pholcidae species on different collecting localities throughout the Amazon Basin is variable; at Floresta Nacional de Caxiuanã, the most abundant pholcid is Carapoia fowleri Huber, 2000 (Bonaldo et al. 2009). This variation could be the result of differences in the sampling effort and collecting methods and/or real differences between the local biota. A summary of the data (number of individuals, collecting method used and frequency of occurrence) for all pholcid species from Porto Urucu is shown in table I. The remaining Pholcidae species had similar abundances by samples, according to the Dunn test results.

The sex ratio in C. ocaina was $1.45\left(\chi^{2}=0.926\right.$, d.f. $=1$, $\mathrm{p}=0.4414$; with Yates correction), in $M$. aurantiacus 1.08 ( $\chi^{2}=0.037$, d.f. $=1, p=1$, with Yates correction) showing that the ratios were fairly $1: 1$ and the differences found are likely due to stochastic variation. In M. taruma the sex ratio was 0.63 $\left(\chi^{2}=4.742\right.$, d.f. $=1, \mathrm{p}=0.0381$, with Yates correction $)$ and for Mesabolivar sp. was $0.35\left(\chi^{2}=9.524\right.$, d.f. $=1, p=0.0034$, with Yates correction). Table II shows a summary of the data used in the chi-square tests. For C. ocaina and M. aurantiacus the statistical tests did not reveal differences in the sex ratio, but the populations of M. taruma and Mesabolivar sp. at Urucu River Basin appear to be female biased. MACHADo et al. (2007a) measured the sex ratio of two Mesabolivar species, and concluded that Mesabolivar forceps Machado et al., 2007 was male biased, which was explained by the higher activity of males than females, making them easier to capture in pit-fall traps (Álvares et al. 2004), while Mesabolivar mairyara Machado et al., 2007 had a homogeneous sex ratio, which was explained only by the low number of adult specimens collected. Our findings should not be explained by the low sampling intensity, neither by the use of pit-fall traps, as all of the four most abundant Pholcidae species were collected with active methods (beating tray and nocturnal hand searches). Thus, this sex ratio pattern can represent the real sex ratio of these species, because all of the visualized individuals were sampled independently of their sex class, although further studies are also required to understand the whole process of sexual ratio in Pholcidae. At least for species of Metagonia, female biased populations have already been reported by HubER \& SCHÜTTE (2009) who captured more than twice as many females than males - Metagonia osa Gertsch, 1986: 8 males, 18 females; Metagonia uvita Huber, 1997: 6 males, 20 females -, with active diurnal hand collecting.

The abundances of C. ocaina and M. aurantiacus were similar in both forest gap types of different regeneration levels, while M. taruma, Mesabolivar sp. and all the pholcid species sampled at the Urucu River Basin together were statistically higher in the poorly regenerated gap type forests (Tab. III). This data shows that pholcid abundance at Porto Urucu can be higher in poorly regenerated areas, where a myriad of effects could be underway: the availability of microhabitats could increase, certain predators might be absent, individuals might find better conditions for establishing their webs, or food availability could be higher. Thus, Pholcidae species can be considered potential ecological indicators of habitat quality, as the species respond differently to environmental conditions, such as the regeneration stage. This response has already been reported: MACHADo et al. (2007a) argued that M. forceps seems to present some degree of tolerance to habitats influenced by

Table I. Summary of the abundance and capture method of the Pholcidae species found at Porto Urucu, Coari, Amazonas, Brazil. (BT) Beating tray, (N) nocturnal hand collecting, (WIN) Winkler extractor, (CF) canopy fogging, (O) occasional sampling, (FO) frequency of occurrence. *"Forest gap type" does not certainly represent the number of individuals collected in the whole basin.

\begin{tabular}{|c|c|c|c|c|c|c|c|c|c|c|c|}
\hline \multirow{2}{*}{ Pholcid species } & \multicolumn{3}{|c|}{ Number of adults } & \multicolumn{2}{|c|}{ Forest gap type* } & \multicolumn{5}{|c|}{ Capture method } & \multirow{2}{*}{ F.O. (\%) } \\
\hline & Males & Females & Total & I-good & II - poor & BT & $\mathrm{CF}$ & $\mathrm{N}$ & $\mathrm{O}$ & WIN & \\
\hline M. taruma & 19 & 13 & 98 & 10 & 17 & 63 & 1 & 33 & 1 & & 66.37 \\
\hline Mesabolivar sp. & & 1 & 56 & 1 & & 14 & & 42 & & & 34.51 \\
\hline C. ocaina & 2 & 5 & 34 & 6 & 2 & 10 & & 22 & 2 & & 23.01 \\
\hline M. aurantiacus & 1 & 1 & 29 & 2 & & 1 & & 28 & & & 18.58 \\
\hline L. dimona & 12 & 13 & 9 & 10 & 17 & 6 & & 3 & & & 5.31 \\
\hline Metagonia beni & 11 & 31 & 9 & 14 & 40 & 3 & & 6 & & & 7.96 \\
\hline Litoporus sp. & 4 & 5 & 2 & 2 & 7 & 1 & & 1 & & & 1.77 \\
\hline Ibotyporanga sp. & 36 & 51 & 1 & 33 & 60 & & & & & 1 & 0.88 \\
\hline Otavaloa sp. & 1 & & 1 & & 1 & & & 1 & & & 0.88 \\
\hline Pholcidae sp. 1 & & 1 & 1 & 1 & & 1 & & & & & 0.88 \\
\hline Pholcidae sp. 2 & 1 & & 1 & 1 & & 1 & & & & & 0.88 \\
\hline
\end{tabular}

ZOOLOGIA 27 (3): 431-439, June, 2010 
Table II. Abundances of the four most common Pholcidae species collected at Porto Urucu, Coari, Amazonas, Brazil, by sex class, forest gap types and the entire Amazon Basin.

\begin{tabular}{|c|c|c|c|c|c|c|c|c|c|c|c|c|}
\hline & \multicolumn{3}{|c|}{ C. ocaina } & \multicolumn{3}{|c|}{ M. aurantiacus } & \multicolumn{3}{|c|}{ M. taruma } & \multicolumn{3}{|c|}{ Mesabolivar sp. } \\
\hline & Males & Females & Total & Males & Females & Total & Males & Females & Total & Males & Females & Total \\
\hline I - Good & 5 & 5 & 10 & 5 & 5 & 10 & 11 & 22 & 33 & 3 & 11 & 14 \\
\hline II - Poor & 11 & 6 & 17 & 9 & 8 & 17 & 25 & 35 & 60 & 10 & 30 & 40 \\
\hline Entire basin & 20 & 14 & 34 & 15 & 14 & 29 & 38 & 60 & 98 & 13 & 43 & 56 \\
\hline
\end{tabular}

Table III. Mann-Whitney test results to verify abundance by forest gap regeneration type for C. ocaina, M. aurantiacus, M. taruma, Mesabolivar sp. and the combined data for all Pholcidae species collected at Porto Urucu, Coari, Amazonas.

\begin{tabular}{lccccc}
\hline & C. ocaina & M. aurantiacus & M. taruma & Mesabolivar sp. & All species \\
\hline I- good (number of individuals/rank sum) & $10 / 142$ & $10 / 119$ & $33 / 1475$ & $14 / 446$ & $80 / 6386$ \\
II - poor (number of individuals/rank sum) & $17 / 209$ & $17 / 181$ & $60 / 2266$ & $40 / 829$ & $144 / 9904$ \\
Mann-Whitney (U) & 51 & 41 & 529 & 121 & 2291 \\
p-value (bilateral) & 0.0858 & 0.0735 & 0.0006 & 0.0002 & $<0.0001$ \\
\hline
\end{tabular}

human activity; and Machado et al. (2007c) reported that Mesabolivar cantharus Machado et al., 2007, M. camussi Machado et al., 2007, M. cavicelatus Machado et al., 2007 were collected in slightly higher numbers in Pinus than in native areas, although the opposite occurred for Tupigea cantareira Machado et al., 2007.

\section{ACKNOWLEDGMENTS}

The authors would like to thank to Bernhard A. Huber, Adalberto J. Santos and three anonymous referees for comments on an earlier version of the manuscript and English review; and to Milena Andrade (UFPA/NAEA), who kindly made the map. This work was supported by the CNPq (LSC, SCD and DFC Ph.D. grants, ABB PQ grant \#307463/2009-5). This work is part of the Rede CTPetro Amazônia - Fundo Setorial do Petróleo (FINEP/CNPq/PETROBRAS) research program and SCD's Ph.D. thesis. LSC, SCD and DFC are Ph.D. students of the Programa de Pós-Graduação em Zoologia, Museu Paraense Emílio Goeldi and Universidade Federal do Pará.

\section{LITERATURE CITED}

Álvares, E.S.S.; E.O. Machado; C.S. Azevedo \& M. De-Maria. 2004. Composition of the spider assemblage in an urban forest reserve in Southeastern Brazil and evaluation of two sampling method protocols of species richness estimates. Revista Ibérica de Aracnologia 10: 185-194.

Álvarez-Padilla, F. \& G. Hormiga. 2008. A protocol for digesting internal soft tissues and mounting spiders for scanning electron microscopy. Journal of Arachnology 35: 538-542.

Astrin, J.J.; B.A. Huber; B. Misof \& C.F.C. KLÜTsCh. 2006. Molecular taxonomy in pholcid spiders (Pholcidae, Araneae): Evaluation of species identification methods using CO1 and 16S rRNA. Zoologica Scripta 35: 441-457.

Astrin, J.J.; B. Misof; B.A. Huber. 2007. The pitfalls of exageration: molecular and morphological evidence suggests Kaliana is a synonym of Mesabolivar (Araneae: Pholcidae). Zootaxa 1646: 17-30.

Ayres, M.; M. Ayres Jr; D.M. Ayres \& A.S. Santos. 2007. BioEstat 5.0: aplicações estatísticas nas áreas das ciências biológicas e médicas. Belém, Sociedade Civil Mamirauá, CNPq, $339 \mathrm{p}$.

Bonaldo, A.B.; L.S. Carvalho; R. Pinto-da-Rocha; A.L.M. Tourinho; L.T. Miglio; D.F. Candianni; N.F. Lo-Man-Hung; N. Abrahim; B.V.B. Rodrigues; A.D. Brescovit; R. Saturnino; N.C. Bastos; S.C. Dias; B.J.F. Silva; J.M.B. Pereira-Filho; C.A. Rheims; S.M. Lucas; D. Polotow; G.R.S. Ruiz \& R. InDicatTI. 2009. Inventario e historia natural dos aracnideos da Floresta Nacional de Caxiuanã, p. 545-588. In: P.L.B. LisBoA (Org.). Caxiuanã: desafios para a conservação de uma Floresta Nacional na Amazônia. Belém, Museu Paraense Emílio Goeldi, 672p.

BRICEÑo, R.D. 1985. Sticky balls in webs of the spider Modisimus sp. (Araneae, Pholcidae). Journal of Arachnology 13: 267269.

Bruvo-Madaric, B.; B.A. Huber; A. Steinacher \& G. Pass. 2005. Phylogeny of pholcid spiders (Araneae: Pholcidae): combined analysis using morphology and molecules. Molecular Phylogenetics and Evolution 37: 661-673.

Coddington, J.A.; C.E. Griswold; D. Silva; D. Peñaranda \& S. Larcher. 1991. Designing and testing sampling protocols to estimate biodiversity in tropical ecosystems, p. 44-60. In: E.C. DudLEY (Ed.). The unity of evolutionary biology. Portland, Dioscorides Press, Proceedings of the Fourth International Congress of Systematic and Evolutionary Biology, 1160p. 
Eberhard, W.G. 1992a. Notes on the ecology and behaviour of Physocyclus globosus (Araneae, Pholcidae). Bulletin of the British Arachnological Society 9 (2): 38-42.

Eвerhard, W.G. 1992b. Web construction by Modisimus sp. (Araneae, Pholcidae). Journal of Arachnology 20: 25-34.

Eberhard, W.G. \& R.D. BRiceño. 1983. Chivalry in pholcid spiders. Behavioral Ecology Sociobiology 13: 189-195.

Eberhard, W.G. \& R.D. Briceño. 1985. Behavior and ecology of four species of Modisimus and Blechroscelis (Araneae, Pholcidae). Revue Arachnologique 6 (1): 29-36.

HöFer, H. \& A.D. Brescovit. 2001. Species and guild structure of a Neotropical spider assemblage (Araneae; Reserva Ducke, Amazonas, Brazil). Andrias 15: 99-120.

Huber, B.A. 1994. Genital morphology, copulatory mechanism and reproductive biology in Psilochorus simoni (Berland, 1911) (Pholcidae; Araneae). Netherlands Journal of Zoology 44 (1-2): 85-99.

Huber, B.A. 1997a. On American 'Micromerys' and Metagonia (Araneae, Pholcidae), with notes on natural history and genital mechanics. Zoologica Scripta 25 (4): 341-363.

Huber, B.A. 1997b. The pholcid spiders of Costa Rica (Aranae: Pholcidae). Revista de Biologia Tropical 45 (4): 1583-1634.

Huber, B.A. 1997c. On the distinction between Modisimus and Hedypsilus (Pholcidae; Araneae), with notes on behavior and natural history. Zoologica Scripta 25 (3): 233-240.

Huber, B.A. 1997d. Evidence for gustatorial courtship in a haplogyne spider (Hedypsilus culicinus: Pholcidae: Araneae). Netherlands Journal of Zoology 47 (1): 95-98.

Huber, B.A. 1998a. Report of some pholcid spiders collected in Guatemala and Honduras (Araneae, Pholcidae). Revue Suisse de Zoologie 105 (1): 49-80.

Huber, B.A. 1998b. Genital mechanics in some neotropical pholcid spiders (Araneae: Pholcidae), with implications for systematics. Journal of Zoology (London) 244: 587-599.

Huber, B.A. 2000. New World pholcid spiders (Araneae: Pholcidae): a revision at generic level. Bulletin of the American Museum of Natural History 254: 1-348.

Huber, B.A. 2001. The pholcids of Australia (Araneae; Pholcidae): taxonomy, biogeography, and relationships. Bulletin of the American Museum of Natural History 260: 1-144.

Huber, B.A. 2005a. Revision and cladistic analysis of the spider genus Carapoia González-Sponga (Araneae: Pholcidae), with descriptions of new species from Brazil's Atlantic forest. Invertebrate Systematics 19: 541-556.

Huber, B.A. 2005b. Sexual selection research on spiders: progress and biases. Biological Reviews 80: 363-385.

Huber, B.A. 2005c. Pholcidae. In: D. Ubick; P. Paquin; P.E. Cushing $\&$ V. Rотн (Eds). Spiders of North America: an identification manual. Gainesville, American Arachnological Society, 377p

Huber, B.A. \& J.J. Astrin. 2009. Increased sampling blurs morphological and molecular species limits: revision of the Hispaniolan endemic genus Tainonia (Araneae, Pholcidae). Invertebrate Systematics 23: 281-300.
Huber, B.A. \& A.D. Brescovit. 2003. Ibotyporanga Mello-Leitão: tropical spiders in Brazilian semi-arid habitats (Araneae: Pholcidae). Insect Systematics and Evolution 34: 15-20.

Huber, B.A. \& W.G. Eberhard. 1997. Courtship, copulation, and genital mechanics in Physocyclus globosus (Araneae, Pholcidae). Canadian Journal of Zoology 74: 905-918.

Huber, B.A.; N. Fischer \& J.J. Astrin. 2010. High level of endemism in Haiti's last remaining forests: revision of Modisimus (Araneae: Pholcidae) on Hispaniola, using morphology and molecules. Zoological Journal of the Linnean Society 158: 244-299.

Huber, B.A. \& A. Schütte. 2009. Preliminary notes on leafdwelling Metagonia spiders (Araneae: Pholcidae) in the Esquinas Rainforest near La Gamba, Costa Rica: leaf preference, mimesis, and web structure. Contributions to Natural History (Bern) 12: 681-697.

Huber, B.A.; A.D. Brescovit \& C.A. Rheims. 2005a. Exaggerated female genitalia in two new spider species (Araneae: Pholcidae), with comments on genital evolution by female choice versus antagonistic coevolution. Insect Systematics and Evolution 36: 285-292.

Huber, B.A.; C.A. Rheims \& A.D. Brescovit. 2005b. Two new species of litter-dwelling Metagonia spiders (Araneae, Pholcidae) document both rapid and slow genital evolution. Acta Zoologica (Stockholm) 86: 33-40.

Huber, B.A.; G.A. Pérez \& R.L.C. Baptista. 2005c. Leptopholcus (Araneae: Pholcidae) in continental America: rare relicts in low precipitation areas. Bonner Zoologische Beiträge 53 (1/2): 99-107.

Huber, B.A. \& J. Wunderlich. 2006. Fossil and extant species of the genus Leptopholcus in the Dominican Republic, with the first case of egg-parasitism in pholcid spiders (Araneae: Pholcidae). Journal of Natural History 40: 2341-2360.

JaPYASSÚ, H.F. \& C.R. MACAgnan. 2004. Fishing for prey: the evolution of a new predatory tactic among spiders (Araneae, Pholcidae). Revista de Etologia 6 (2): 79-94.

Levi, H.W. 1965. Techniques for the study of spider genitalia. Psyche 72: 152-158.

Machado, E.O.; A.D. Brescovit; D.F. Candiani \& B.A. Huber. 2007 a. Three new species of Mesabolivar González-Sponga, 1998 (Araneae: Pholcidae) from leaf litter in urban environments in the city of São Paulo, São Paulo, Brazil. Iheringia, Série Zoologia, 97 (2): 168-176.

Machado, E.O.; A.D. Brescovit \& R.C. Francisco. 2007b. Three new ground living Mesabolivar González-Sponga 1998 (Araneae: Pholcidae) from Blumenau, Santa Catarina, Brazil. Zootaxa 1560: 55-61.

Machado, E.O.; F. Yamamoto; A.D. Brescovit \& B.A. Huber. 2007c. Three new ground living pholcid species (Araneae: Pholcidae) from Parque Estadual da Cantareira, São Paulo, São Paulo, Brazil. Zootaxa 1582: 27-37.

Peretti, A.; W.G. Eberhard \& R.D. Briceño. 2006. Copulatory dialogue: female spiders sing during copulation to influence male genitalic movements. Animal Behavior 72: 413-421. 
Pérez-González, A. \& B.A. Huber. 1999. Metagonia debrasi n. sp., the first species of the genus Metagonia Simon in Cuba (Pholcidae, Araneae). Revue Arachnologique 13 (4): 69-72.

Platnick, N.I. 2009. The World Spider Catalog. Version 9.5. American Museum of Natural History. Available online at: http://research.amnh.org/entomology/spiders/catalog/index. html [Accessed: 5.VII.2009].

RADAM-BRASIL. 1978. Programa de Integração Nacional. Levantamentos de Recursos Naturais. Manaus, Ministério das Minas e Energia, vol. 18, 626p.

Ribeiro; J.E.L.S.; M.J.G. Hopkins; A. Vicentini; C.A. Sothers; M.A.S. Costa; J.M. Brito; M.A. Sousa; L.H.P. Martins; L.G. Lohmann; P.A.C.L. Assunção; E.C. Pereira; C.F. Silva; M.R. Mesquita \&
L.C. Procópio. 1999. Flora da Reserva Ducke: Guia de identificação das plantas vasculares de uma floresta de terrafirme na Amazônia Central. Manaus, Instituto Nacional de Pesquisas da Amazônia, 799p.

Sewlal, J.N. \& C.K. Starr. 2008. Observations of the insect Arachnocoris trinitatis (Heteroptera: Nabidae) as an inquiline of the spider Mesabolivar aurantiacus (Araneae: Pholcidae). Caribean Journal of Science 44: 132-135.

Silva, D. \& J.A. Coddington. 1996. Spiders of Pakitza (Madre de Dios, Perú): species richness and notes on community structure, p. 253-311. In: D.E. Wilson \& A. SANDoval (Eds). Manu-The biodiversity of Southeastern Peru. Washington, D.C., Smithsonian Institution Press, 679p.

Submitted: 31.VII.2009; Accepted: 10.IV.2010.

Editorial responsibility: Antonio Domingos Brescovit 